\title{
The human genome is ten years old: has it been a success?
}

\author{
Marco Bregni
}

Received: 9 June 2010/Accepted: 18 October 2010/Published online: 16 November 2010

(C) Springer-Verlag 2010

Dear Editor,

In June 2000, the announcement of the first complete sequencing of the human genome was made by two companies, the international, publicly funded Human Genome Project and the private, for-profit US company Celera Genomics, in the presence of the US President Bill Clinton and the UK Prime Minister Tony Blair. This event was recalled in a recent issue of Nature [1] with several articles from people who were involved in that enterprise. The question asked to those people was the following: despite all the intellectual ferment of the past decade, has human health truly benefited from the sequencing of the human genome? This question is highly relevant, both for the giant investments that have been made, and for the hope that this result has generated in patients and in healthcare workers. The startlingly honest response from the leaders of the public and private efforts, Francis Collins and Craig Venter, was 'Not much'. Granted, there has been some progress, in the form of drugs targeted against specific genetic defects identified in a few types of cancer, and in some rare inherited disorders. But the complexity of post-genome biology has dashed early hopes that this trickle of therapies would rapidly become a flood. Witness the multitude of association studies that aimed to find connections between common genetic variants and common diseases, with only limited success, or the discovery that most cancers have their own unique genetic characteristics, making widely applicable therapies hard to find. Among the other comments, the article by Robert Weinberg, professor of biology at MIT, addressed the particularly relevant issue of research methodology in the post-genomic era [2]. Weinberg is a top cancer scientist, discoverer of

M. Bregni ( $\square)$

Osp. San Giuseppe, Via San Vittore 12, 20123 Milan, Italy

e-mail: marco.bregni@hsr.it mechanisms of tumorigenesis and of several oncogenes (ras, amongst others) [3, 4]. In his opinion, biology-traditionally a descriptive science-has became one of hypothesis-driven experimentation. Tightly coupled with this was the increasing dominance of reductionism, the idea that complex biological systems can be understood by dismantling them into their constituent pieces and studying each in isolation. These approaches over the past half-century have witnessed the revolutions in molecular and cellular biology, immunology, neurobiology and genetics. Now, the dominant position of hypothesis-driven research is under threat. Large-scale efforts such as sequencing of entire tumour genomes, or their coding exons, consume an enormous amount of resources and researchers' energy. The dividends to date have been modest: the discovery of some new oncogenes and tumour suppressor genes involved in tumour formation (e.g., BRAF, IDH1/2 and translocations in prostate carcinomas), and a general measure of the degree of genetic instability of various tumour-cell genomes. Is it worthwhile to abandon hypothesis-driven research and divert all funds towards massive data-generating projects? The question is relevant, as the education and the professional behaviour of new generations of scientists and clinical investigators will depend on it. In our opinion, the beauty of research lies in formulating hypotheses and trying to solve them: in other words, in the direct involvement of the investigator as a person.

\section{References}

1. Editorial (2010) The human genome at ten. Nature 464:649

2. Weinberg RA (2010) Point: hypotheses first. Nature 464:678

3. Weinberg RA (2008) Mechanisms of malignant progression. Carcinogenesis 29:1092-1095

4. Karnoub AE, Weinberg RA (2008) Ras oncogenes: split personalities. Nat Rev Mol Cell Biol 9:517-531 